OPEN ACCESS

Edited by:

Jo Corbett,

University of Portsmouth, UK

Reviewed by:

José González-Alonso,

Brunel University London, UK

Ana Sousa,

Universidade de Trás-os-Montes e

Alto Douro, Portugal

${ }^{*}$ Correspondence:

Camila C. Greco

grecocc@rc.unesp.br

Specialty section:

This article was submitted to

Exercise Physiology,

a section of the journal

Frontiers in Physiology

Received: 24 August 2016 Accepted: 18 November 2016 Published: 02 December 2016

Citation:

Barbosa LF, Denadai BS and

Greco CC (2016) Endurance

Performance during Severe-Intensity Intermittent Cycling: Effect of Exercise

Duration and Recovery Type. Front. Physiol. 7:602

doi: 10.3389/fphys.2016.00602

\section{Endurance Performance during Severe-Intensity Intermittent Cycling: Effect of Exercise Duration and Recovery Type}

\author{
Luis F. Barbosa, Benedito S. Denadai and Camila C. Greco* \\ Human Performance Laboratory, Biosciences Institute, São Paulo State University, Rio Claro, Brazil
}

Slow component of oxygen uptake $\left(\mathrm{VO}_{2} \mathrm{SC}\right)$ kinetics and maximal oxygen uptake $\left(\mathrm{VO}_{2}\right.$ max) attainment seem to influence endurance performance during constant-work rate exercise (CWR) performed within the severe intensity domain. In this study, it was hypothesized that delaying the attainment of $\mathrm{VO}_{2}$ max by reducing the rates at which $\mathrm{VO}_{2}$ increases with time $\left(\mathrm{VO}_{2} \mathrm{SC}\right.$ kinetics) would improve the endurance performance during severe-intensity intermittent exercise performed with different work:recovery duration and recovery type in active individuals. After the estimation of the parameters of the $\mathrm{VO}_{2} \mathrm{SC}$ kinetics during CWR exercise, 18 males were divided into two groups (Passive and Active recovery) and performed at different days, two intermittent exercises to exhaustion (at $95 \% \mathrm{IVO}_{2} \max$, with work: recovery ratio of 2:1) with the duration of the repetitions calculated from the onset of the exercise to the beginning of the $\mathrm{VO}_{2} \mathrm{SC}$ (Short) or to the half duration of the $\mathrm{VO}_{2} \mathrm{SC}$ (Long). The active recovery was performed at $50 \% \mathrm{IVO}_{2}$ max. The endurance performance during intermittent exercises for the Passive (Short $=1523$ \pm 411 ; Long $=984 \pm 260 \mathrm{~s}$ ) and Active (Short = $902 \pm 239$; Long = $886 \pm 254 \mathrm{~s}$ ) groups was improved compared with CWR condition (Passive $=540 \pm 116$; Active $=$ $489 \pm 84 \mathrm{~s}$ ). For Passive group, the endurance performance was significantly higher for Short than Long condition. However, no significant difference between Short and Long conditions was found for Active group. Additionally, the endurance performance during Short condition was higher for Passive than Active group. The $\mathrm{VO}_{2} \mathrm{SC}$ kinetics was significantly increased for CWR (Passive $=0.16 \pm 0.04$; Active $=0.16 \pm 0.04$ L. min $^{-2}$ ) compared with Short (Passive $=0.01 \pm 0.01$; Active $=0.03 \pm 0.04$ L. $\mathrm{min}^{-2}$ ) and Long (Passive $=0.02 \pm 0.01$; Active $=0.01 \pm 0.01 \mathrm{~L} \cdot \mathrm{min}^{-2}$ ) intermittent exercise conditions . No significant difference was found among the intermittent exercises. It can be concluded that the endurance performance is negatively influenced by active recovery only during shorter high-intensity intermittent exercise. Moreover, the improvement in endurance performance seems not be explained by differences in the $\mathrm{VO}_{2} \mathrm{SC}$ kinetics, since its values were similar among all intermittent exercise conditions.

Keywords: aerobic, oxygen uptake, passive, active, exercise tolerance 


\section{INTRODUCTION}

The parameters of the power-time relationship, termed critical power $(\mathrm{CP})$ and the curvature constant (W'), have been used to analyze the physiological responses and endurance performance during high-intensity exercise (Poole et al., 1988). CP has been considered the lower boundary of the severe-intensity domain and the $\mathrm{W}^{\prime}$ determines the amount of external work that can be performed above $\mathrm{CP}$, irrespective of the rate of its expenditure (Jones et al., 2010). By definition, all severe-intensity work rates (i.e., $>\mathrm{CP}$ ) performed until voluntary exhaustion drive pulmonary oxygen uptake $\left(\mathrm{VO}_{2}\right)$ to a maximal value (i.e., maximal oxygen uptake- $\mathrm{VO}_{2} \max$ ) (Jones et al., 2010). However, during exhaustive exercise performed above the upper bound of the severe intensity domain, exercise duration would be too short to permit attainment of $\mathrm{VO}_{2}$ max Caputo and Denadai (2008). Several studies have demonstrated that endurance exercise performance within severe-intensity domain was coincident with the depletion of the W', accumulation of metabolites associated with fatigue (i.e., $\mathrm{PCr}, \mathrm{Pi}$, and $\mathrm{H}^{+}$), and attainment of $\mathrm{VO}_{2} \max$ due to $\mathrm{VO}_{2}$ slow component $\left(\mathrm{VO}_{2} \mathrm{SC}\right.$ ) development (Fukuba et al., 2003; Chidnok et al., 2013). Indeed, $\mathrm{VO}_{2} \mathrm{SC}$ has been associated with loss in muscular efficiency (Jones et al., 2011) and has been negatively related with endurance performance (Zoladz et al., 1995; Murgatroyd et al., 2011; Barbosa et al., 2014a).

$\mathrm{VO}_{2}$ kinetics and muscle [PCr] responses to high-intensity exercise have been reported to present both fundamental and slow component phases (Rossiter et al., 2002) being intrinsically linked. Indeed, Rossiter et al. (2002) have reported similar values of the time constant $(\tau)$ of the fundamental component $\left([\mathrm{PCr}]=38 \mathrm{~s} ; \mathrm{VO}_{2}=39 \mathrm{~s}\right)$, as well as the relative amplitude of the slow component $\left([\mathrm{PCr}]=13.9 \% ; \mathrm{VO}_{2} \mathrm{SC}=15.3 \%\right)$ of muscle $[\mathrm{PCr}]$ and $\mathrm{VO}_{2}$ during high-intensity exercise. It has been proposed that progressive intramuscular depletion $[\mathrm{PCr}]$ during exhaustive exercise performed within severe intensity domain provides the appropriate stimulus to oxidative phosphorylation, determining the development of $\mathrm{VO}_{2} \mathrm{SC}$ and, consequently, the attainment of $\mathrm{VO}_{2} \max$ (Rossiter et al., 2002). Thus, both creatine phosphate depletion and development of the $\mathrm{VO}_{2} \mathrm{SC}$ seem to be intimately associated with endurance performance during constant-work rate exercise (CWR) performed within the severe intensity domain.

While this scenario is well established during CWR exercise, very little information is available during intermittent exercise, which has been considered an important tool in training programs aiming to improve aerobic fitness in health and in disease (Laursen and Jenkins, 2002; Hwang et al., 2011). Indeed, intermittent exercise can improve performance comparing to CWR during high-intensity exercise (Millet et al., 2003; Chidnok et al., 2012), since the former allows resynthesis of intramuscular substrates ([PCr] $)$ and/or clearance of fatigue-related metabolites (i.e., reconstitution of W') (Chidnok et al., 2013). However, several aspects seem to influence endurance performance during high-intensity intermittent exercises. For instance, endurance performance is progressively shorter when the work-recovery "duty-cycle" (e.g., 10:20 s, 30:60 s, 60:120 s, and 90:180 s) (Turner et al., 2006) and/or exercise intensity performed during active recovery is increased (i.e., light, moderate, heavy and severe) (Chidnok et al., 2012). These aspects influence PCr kinetics (Chidnok et al., 2013) and hypothetically, the changes of the rates at which $\mathrm{VO}_{2}$ increases during high-intensity intermittent exercises (i.e., $\mathrm{VO}_{2} \mathrm{SC}$ ). Indeed, Chidnok et al. (2012) have demonstrated that enhanced endurance performance during severe-intensity intermittent exercise could be explained by the reconstitution of $\mathrm{W}^{\prime}$ during recovery intervals performed at lower-intensity domains (i.e., light and moderate). At this condition, the reconstitution of $\mathrm{W}^{\prime}$ was associated with a blunted increase in both $\mathrm{VO}_{2}$ and integrated EMG with time, supporting the hypothesis that $\mathrm{VO}_{2} \mathrm{SC}$ kinetics influences endurance performance during intermittent exercise. However, as discussed above, endurance performance during severe intermittent exercise is markedly modulated by both workrecovery duration and exercise intensity performed during active recovery. Thus, the possible relationship between $\mathrm{VO}_{2} \mathrm{SC}$ and endurance performance during intermittent exercise performed with different durations (e.g., short vs. long) and recovery type (i.e., passive vs. active) remains elusive, and further studies are warranted.

However, an important issue must be considered when the possible influence of $\mathrm{VO}_{2} \mathrm{SC}$ on endurance performance is investigated. Knowing that work-recovery duration influences endurance performance during severe intermittent exercise (Turner et al., 2006), it appears appropriate to compare exercise duration before (short condition) and after (long condition) the emergence of $\mathrm{VO}_{2} \mathrm{SC}$. However, many studies have verified that both the emergence and the amplitude of $\mathrm{VO}_{2} \mathrm{SC}$ (and possibly the $[\mathrm{PCr}]$ ) present a large intra-individual variation (Murgatroyd et al., 2011; Barbosa et al., 2014b). Thus, it would be interesting to analyze the responses of $\mathrm{VO}_{2}$ kinetics and endurance performance during severe intermittent exercise, with both the duration of exercise and recovery periods being determined based on the individual $\mathrm{VO}_{2} \mathrm{SC}$ kinetics response.

Thus, the current study was undertaken to compare the endurance performance and $\mathrm{VO}_{2} \mathrm{SC}$ kinetics during highintensity intermittent exercise performed with different work:recovery duration (short vs. long) and recovery types (passive vs. active) in active individuals. It was hypothesized that: (a) endurance performance would be improved during the exercise with passive recovery, regardless of the duration of the repetition, and; (b) endurance performance would be improved during the intermittent exercise with short duration, regardless of the recovery type. We also hypothesized that the possible interaction between exercise duration and recovery type during intermittent high intensity exercise would influence the changes to the rates at which $\mathrm{VO}_{2}$ increases with time $\left(\mathrm{VO}_{2} \mathrm{SC}\right.$ kinetics $)$ and consequently, endurance performance.

\section{MATERIALS AND METHODS}

\section{Subjects}

Eighteen male students $(24.7 \pm 4.1$ years; $80.5 \pm 12.5 \mathrm{~kg} ; 178.1$ $\pm 7.6 \mathrm{~cm}$ ) that were physically active but did not participate in any regular physical exercise or sport program volunteered for 
the study. All participants were healthy and free of cardiovascular, respiratory, and neuromuscular disease. All risks associated with the experimental procedures were explained prior to involvement in the study and each participant signed an informed consent form. The study was performed according to the Declaration of Helsinki and the protocol was approved by the University's Ethics Committee.

\section{Experimental Design}

The participants were instructed to report to the laboratory at the same time of the day $( \pm 2 \mathrm{~h})$ on four separate occasions within a period of 2-3 week. Firstly, each volunteer performed an incremental test until exhaustion to determine the lactate threshold (LT), $\mathrm{VO}_{2} \max$ and the intensity associated with $\mathrm{VO}_{2} \max \left(\mathrm{IVO}_{2} \mathrm{max}\right)$. Thereafter, the volunteers were divided into two groups: passive recovery (PR) and active recovery (AR) with similar $\mathrm{IVO}_{2} \max$ values. They performed the following protocols, on different days: (1) a total of two repetitions of square-wave transitions from rest to a power corresponding to $95 \%$ of the $\mathrm{IVO}_{2}$ max to determine the parameters of $\mathrm{VO}_{2}$ kinetics. Each bout was separated by $60 \mathrm{~min}$ of passive rest. The $\mathrm{VO}_{2}$ responses to the two severe exercise bouts were averaged before the analysis to reduce the breath-to-breath noise and enhance confidence in the parameters derived from the modeling process (Lamarra et al., 1987) and; (2) two intermittent exercises, with the duration of the repetitions calculated from the onset of the exercise to the beginning of the $\mathrm{VO}_{2} \mathrm{SC}$ (Short) or to the half duration of the $\mathrm{VO}_{2} \mathrm{SC}$ (Long). The interval between the experimental sessions was $48-72 \mathrm{~h}$. The participants were instructed to arrive at the laboratory in a rested and fully hydrated state at least $3 \mathrm{~h}$ post-prandial. They were also asked not to perform any strenuous activity during the day before each test.

\section{Procedures}

\section{Incremental Test}

Each participant performed an incremental exercise test to obtain volitional fatigue on an electronically braked cycle ergometer (Excalibur sport, Groningen, Netherlands) to determine the participant's LT, $\mathrm{VO}_{2} \max$, and $\mathrm{IVO}_{2} \max$. The incremental protocol started at a power output of $35 \mathrm{~W}$, with increasing increments of $35 \mathrm{~W}$ every $3 \mathrm{~min}$. Previous studies have demonstrated no differences in $\mathrm{VO}_{2}$ max between incremental tests involving 1- or 3-min stage durations (Bentley and McNaughton, 2003; Roffey et al., 2007; Adami et al., 2013). The pedal cadence was kept constant (70 rpm) (Marsh and Martin, 1997). Throughout the tests, the respiratory and pulmonary gasexchange variables were measured using a breath-by-breath gas analyzer (Quark PFTergo, Cosmed, Italy). The $\mathrm{VO}_{2}$ max was defined as the highest average $15-\mathrm{SO}_{2}$ value recorded during the incremental test. $\mathrm{IVO}_{2}$ max was defined as the power output at which the $\mathrm{VO}_{2}$ max occurred. At the end of each stage, an earlobe capillary blood sample $(25 \mu \mathrm{L})$ was collected into an eppendorf tube and analyzed for its lactate concentration ([La]) using an automated analyzer (YSI 2300 STAT, Yellow Spring, Ohio, USA). Plots of the blood [La] against the power output and $\mathrm{VO}_{2}$ were given to two independent reviewers, who determined LT as the first sudden and sustained increase in the blood lactate level above the resting concentrations.

\section{Constant-Workload Exercise}

The participants performed two exercise transitions at $95 \%$ $\mathrm{IVO}_{2}$ max, separated by $60 \mathrm{~min}$ of rest. The first transition lasted $6 \mathrm{~min}$ and was conducted to determine the $\mathrm{VO}_{2}$ kinetics. The second transition was conducted until voluntary exhaustion to determine the $\mathrm{VO}_{2}$ kinetics (first $6 \mathrm{~min}$ ) and the tlim (time to exhaustion). The protocol began with a $5 \mathrm{~min}$ warm-up at $50 \% \mathrm{IVO}_{2} \max$ and was followed by a $7 \mathrm{~min}$ of passive rest. Then, the participants performed $3 \mathrm{~min}$ of unloaded cycling at $20 \mathrm{~W}$, followed by a step change in the power output to $95 \% \mathrm{IVO}_{2}$ max. The pedal cadence was kept constant at $70 \mathrm{rpm}$. The second transition was terminated when the participant could not maintain a cadence of $>65 \mathrm{rpm}$ for $>5 \mathrm{~s}$ despite verbal encouragement. The end-exercise $\mathrm{VO}_{2}$ was defined as the mean $\mathrm{VO}_{2}$ measured during the final $15 \mathrm{~s}$ of exercise. For the determination of [La] peak, capillary blood samples were collected 1,3 , and $5 \mathrm{~min}$ after the exercise, as previously described.

\section{Intermittent Exercises}

The intermittent exercises were performed at $95 \% \mathrm{IVO}_{2} \mathrm{max}$, with the duration of the repetitions calculated from the onset of the exercise to the beginning of the $\mathrm{VO}_{2} \mathrm{SC}$ (i.e., time delay before the onset of the development of the $\mathrm{VO}_{2} \mathrm{SC}-\mathrm{Short}$ ) or the half duration of the $\mathrm{VO}_{2} \mathrm{SC}$ (i.e., $50 \%$ of the difference between the Short work interval duration and the time to achieve $\mathrm{VO}_{2} \mathrm{max}$-Long) (Figure 1). The recovery was passive (PR) or active (AR) (50\% $\left.\mathrm{IVO}_{2} \mathrm{max}\right)$, with duration corresponding to the half of the repetition (effort:recovery ratio of 2:1). The exercises were performed until voluntary exhaustion. The criterion of exhaustion used was the same used for the constant-workload exercise. The end-exercise $\mathrm{VO}_{2}$ was defined as the mean $\mathrm{VO}_{2}$ measured during the final $15 \mathrm{~s}$ of exercise. If the duration of the last repetition was shorter than $90 \mathrm{~s}$, the highest value of the previous bout was considered, to avoid underestimating the $\mathrm{VO}_{2}$ value.

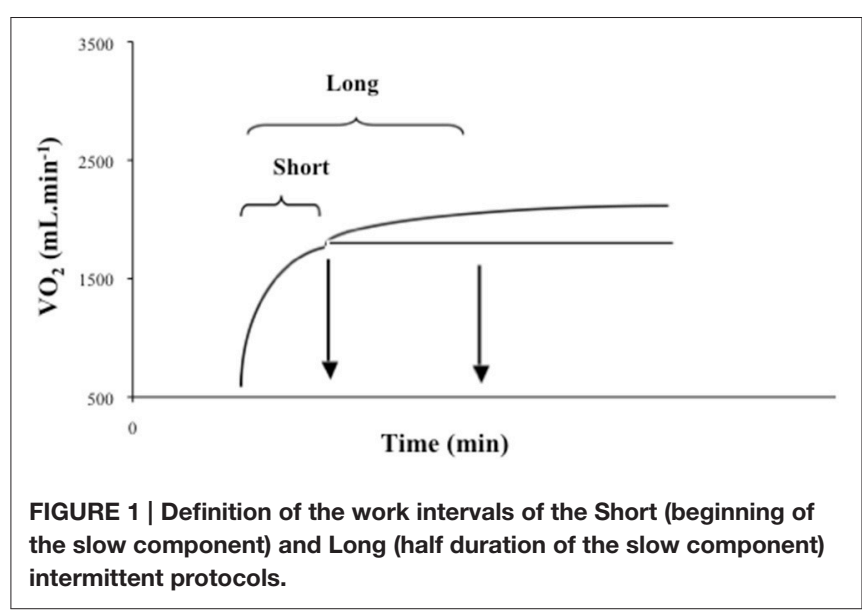




\section{Modeling of $\mathrm{VO}_{2}$ during Constant-Workload Exercise} The breath-by-breath data from each exercise were manually filtered to remove outlying breaths, which were defined as breaths $\pm 3 \mathrm{SD}$ from the adjacent five breaths. The breath-by-breath data were interpolated to give second-by-second values. For CWR, the two transitions were then time aligned to the start of the exercise and averaged to enhance the underlying response characteristics. The first $20 \mathrm{~s}$ of data after the onset of exercise (i.e., the phase I response) (Whipp and Rossiter, 2005) were deleted, and the biexponential model was used to analyze the $\mathrm{VO}_{2}$ response to severe exercise, as described by the following equation:

$$
\begin{aligned}
\mathrm{VO}_{2}(\mathrm{t})= & \mathrm{VO}_{2} \text { baseline }+\mathrm{Ap}\left[1-\mathrm{e}^{-(\mathrm{t}-\mathrm{TDp}) /} \tau^{\mathrm{p}}\right] \\
& + \text { As }\left[1-\mathrm{e}^{-(\mathrm{t}-\mathrm{TDs}) /} \tau^{\mathrm{s}}\right]
\end{aligned}
$$

where: $\mathrm{VO}_{2}(\mathrm{t})$ is the absolute $\mathrm{VO}_{2}$ at a given time $\mathrm{t}$; $\mathrm{VO}_{2}$ baseline is the mean $\mathrm{VO}_{2}$ in the baseline period; $\mathrm{Ap}, \mathrm{TDp}$, and $\tau \mathrm{p}$ are the amplitude, time delay, and time constant, respectively, describing the phase II increase in $\mathrm{VO}_{2}$ above baseline; and As, TDs, and $\tau$ s are the amplitude of, time delay before the onset of, and time constant describing the development of the $\mathrm{VO}_{2} \mathrm{SC}$, respectively. An iterative process was used to minimize the sum of the squared errors between the fitted function and the observed values. $\mathrm{VO}_{2}$ baseline was defined as the mean $\mathrm{VO}_{2}$ measured over the final $60 \mathrm{~s}$ of exercise preceding the step transition to severe exercise. The amplitude of the $\mathrm{VO}_{2} \mathrm{SC}$ was determined as the increase in $\mathrm{VO}_{2}$ from TDs to the end of the modeled data (defined as As'). The end-exercise $\mathrm{VO}_{2}$ was defined as the mean $\mathrm{VO}_{2}$ measured over the final $15 \mathrm{~s}$ of exercise. The TD identified from Equation 1 was utilized to individualize the duration of the repetitions performed during short and long protocols (please see Section Intermittent exercises) and to estimate the $\mathrm{VO}_{2} \mathrm{SC}$ kinetics [i.e., the slow component trajectory $\left(\mathrm{L} \cdot \mathrm{min}^{-2}\right)$ ], as described below.

In addition, a single-exponential model without time delay, with a fitting window commencing at $t=0 \mathrm{~s}$ (equivalent to the mean response time), was used to characterize the kinetics of the overall $\mathrm{VO}_{2}$ response to exercise. The following equation describes this model:

$$
\mathrm{VO}_{2}(\mathrm{t})=\mathrm{VO}_{2 \text { baseline }}+\mathrm{A}\left[1-\mathrm{e}^{-(\mathrm{t} /} \tau^{)}\right]
$$

where: $\mathrm{VO}_{2}(\mathrm{t})$ represents the absolute $\mathrm{VO}_{2}$ at a given time $\mathrm{t}$, $\mathrm{VO}_{2}$ baseline represents the mean $\mathrm{VO}_{2}$ measured over the final $60 \mathrm{~s}$ of baseline pedaling, and $\mathrm{A}$ and $\tau$ represent the amplitude and time constant, respectively, which describe the overall increase in $\mathrm{VO}_{2}$ above the baseline. The $\mathrm{VO}_{2}$ was assumed to have essentially reached its maximal value when the value of $\left[1-\mathrm{e}^{-(t / \tau)}\right]$ from Equation 2 was 0.99 (i.e., when $\left.t=4.6 \times \tau\right)$; it was assumed at this time that $\mathrm{VO}_{2}$ was at its maximal value. Therefore, for each exercise, the time to achieve $\mathrm{VO}_{2} \max$ $\left(\mathrm{TAVO}_{2} \mathrm{max}\right)$ was defined as $4.6 \times \tau$. $\mathrm{VO}_{2} \mathrm{SC}$ kinetics [i.e., the slow component trajectory $\left(\mathrm{L} . \mathrm{min}^{-2}\right.$ )] was also estimated by calculating the slope of the $\mathrm{VO}_{2}$ response using linear regression analysis (Chidnok et al., 2012). The data obtained before TDs (determined from Equation 1) were deleted to remove the influence of the fundamental response phase, and thereafter,
$\mathrm{VO}_{2}$ values at 60 -s intervals were determined until reaching the $\mathrm{TAVO}_{2}$ max value and were fitted using the following equation:

$$
\mathrm{VO}_{2}=a x+b
$$

where: $\mathrm{x}$ represents the time, a represents the slope, and $\mathrm{b}$ represents the $y$-intercept.

\section{Modeling of $\mathrm{VO}_{2}$ during Intermittent Exercise}

$\mathrm{VO}_{2} \mathrm{SC}$ kinetics [i.e., the slow component trajectory $\left(\mathrm{L} . \mathrm{min}^{-2}\right)$ ] was estimated by calculating the slope of $\mathrm{VO}_{2}$ response using linear regression analysis (Chidnok et al., 2012). Final $\mathrm{VO}_{2}$ values (i.e., the average $\mathrm{VO}_{2}$ during $15 \mathrm{~s}$ ) of each work cycle during intermittent exercise were determined up to the last completed cycle and fit using the Equation 3.

\section{Statistical Analysis}

The data are presented as means \pm SD. The normality of data was checked by the Shapiro-Wilk test. A $2 \times 3$ two-way factorial analysis of variance (group vs. exercise condition), with repeated measures for the exercise condition factor (CWR vs. Short vs. Long) was used to analyze the $\mathrm{VO}_{2}$, tlim, slope $\mathrm{VO}_{2}$, [La] and HR data. When a significant interaction was found, follow-up analyses were performed using Tukey HSD test. The significance level was set at $p<0.05$, and effect sizes were calculated using partial eta-squared $\left(\eta^{2}\right)$. All analyses were completed using the Statistical Package for the Social Sciences (SPSS v.20.0, SPSS Inc., Chicago, IL, USA).

\section{RESULTS}

Table 1 presents the mean \pm SD values of the variables obtained during the incremental test for both PR and AR groups. No significant difference was found between the groups $(p>0.05)$.

The $\mathrm{VO}_{2}$ response profiles of a representative subject obtained during the different exercise conditions for both $\mathrm{PR}$ and $\mathrm{AR}$ groups are depicted in Figure 2. Based on the $\mathrm{VO}_{2}$ kinetics parameters obtained during CWR, the repetition duration for the Short $(\mathrm{PR}=105 \pm 29 \mathrm{~s} ; \mathrm{AR}=132 \pm 39 \mathrm{~s})$ and Long $(\mathrm{PR}=252$ $\pm 50 \mathrm{~s} ; \mathrm{AR}=253 \pm 56 \mathrm{~s}$ ) tests were not significantly different between the groups $(p>0.05)$.

Figure 3 presents the mean $\pm \mathrm{SD}$ values of end-exercise $\mathrm{VO}_{2}$ measured during the different exercise conditions for both PR and AR groups. There was a significant main effect for the

TABLE 1 | Mean \pm SD values of the variables obtained during the incremental test for both passive (PR) and active (AR) recovery groups.

\begin{tabular}{lcc}
\hline & PR $(\boldsymbol{N}=\mathbf{9})$ & AR $(\boldsymbol{N}=\mathbf{9})$ \\
\hline $\mathrm{VO}_{2} \max \left(\mathrm{mL} \cdot \mathrm{min}^{-1}\right)$ & $3220.4 \pm 271.8$ & $3332.4 \pm 499.1$ \\
$\mathrm{IVO}_{2} \max (\mathrm{W})$ & $250.3 \pm 25.5$ & $266.9 \pm 44.1$ \\
$\mathrm{P} 95 \%(\mathrm{~W})$ & $235.7 \pm 23.0$ & $252.6 \pm 42.7$ \\
$\mathrm{LT}(\mathrm{W})$ & $106.0 \pm 31.3$ & $133.1 \pm 59.0$ \\
$\mathrm{LT}\left(\% \mathrm{IVO}_{2}\right.$ max & $41 \pm 11$ & $48 \pm 16$
\end{tabular}

$\mathrm{VO}_{2}$ max, maximal oxygen uptake; $\mathrm{VO}_{2}$ max, intensity at $\mathrm{VO}_{2}$ max; $P 95 \%$, power output relative to $95 \% \mathrm{NVO}_{2}$ max; $L T$, lactate threshold. 


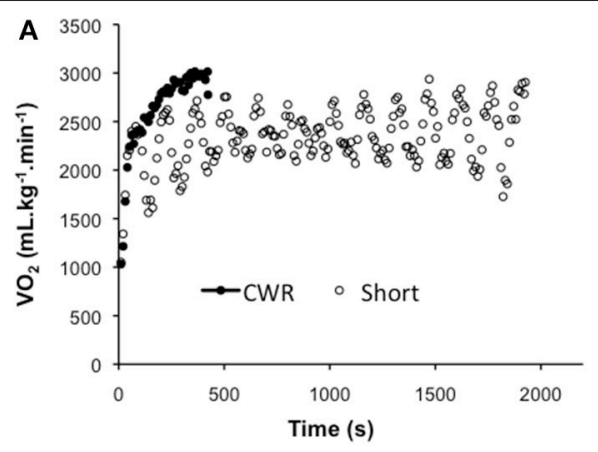

B
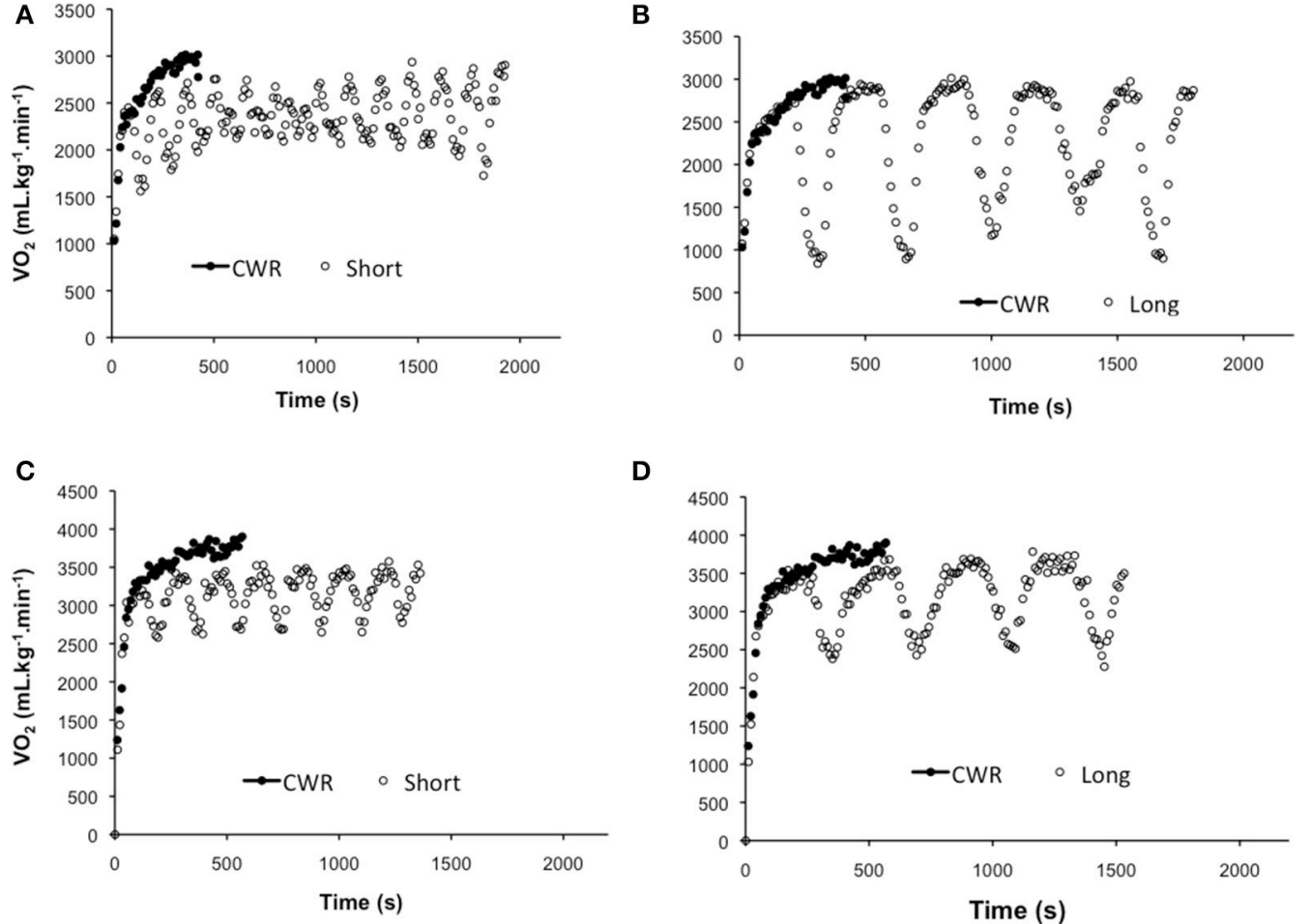

D

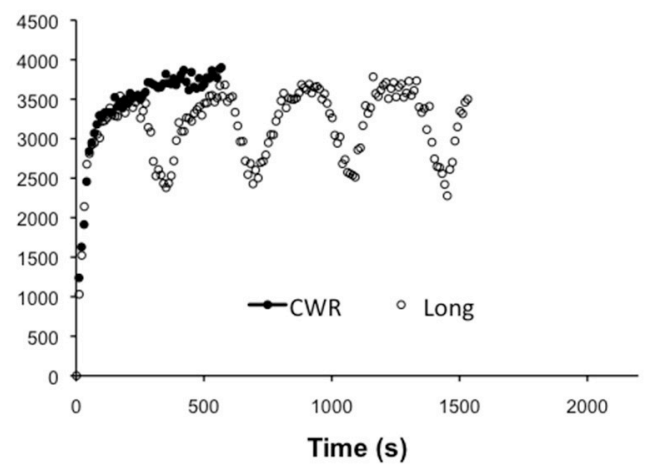

FIGURE 2 | Pulmonary oxygen uptake $\left(\mathrm{VO}_{2}\right)$ response of a representative subject to constant-work rate (CWR) exercise (closed circles) compared with intermittent exercise (open circles) performed with passive (A, short and $\mathbf{B}$, long) and active (C, short and $\mathbf{D}$, long) recovery.

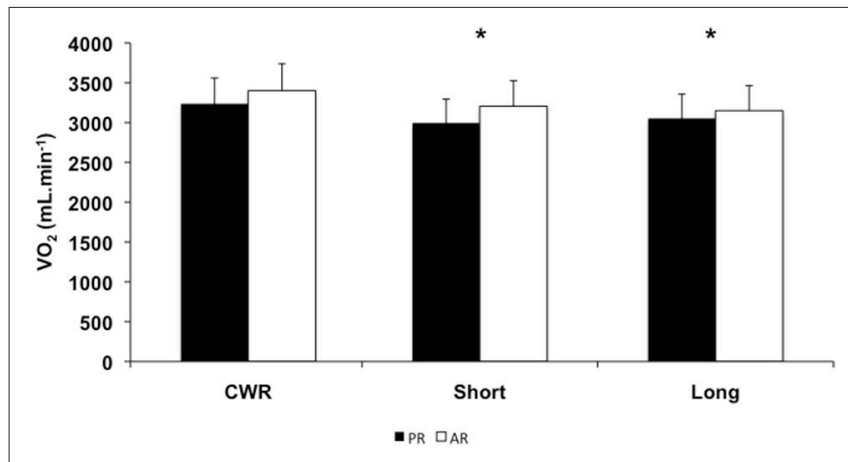

FIGURE 3 | Mean \pm SD values of the end-exercise $\mathrm{VO}_{2}$ obtained during the exercise performed in different conditions for passive (PR) $(N=9)$ and active (AR) $(\mathbf{N}=\mathbf{9})$ groups. CWR-constant-work-rate exercise; ${ }^{*} p<$ 0.05 in relation to CWR.

exercise condition on end-exercise $\mathrm{VO}_{2}$ values $(F=5.47, p=$ $\left.0.009, \eta^{2}=0.25\right)$, but no effect of group $\left(F=1.53, p=0.23, \eta^{2}\right.$ $=0.08)$ or interaction was detected $\left(F=1.25, p=0.29, \eta^{2}=\right.$ 0.07). The end-exercise $\mathrm{VO}_{2}$ values obtained during CWR (PR $\left.=3236.9 \pm 405.8 \mathrm{~mL} \cdot \mathrm{min}^{-1} ; \mathrm{AR}=3488.6 \pm 415.9 \mathrm{~mL} \cdot \mathrm{min}^{-1}\right)$ were higher than those attained during Short $(\mathrm{PR}=2995.2 \pm$ $\left.337.7 \mathrm{~mL} \cdot \mathrm{min}^{-1} ; \mathrm{AR}=3205.7 \pm 447.2 \mathrm{~mL} \cdot \mathrm{min}^{-1}\right)$ and Long (PR $\left.=3053.3 \pm 276.1 \mathrm{~mL} \cdot \mathrm{min}^{-1} ; \mathrm{AR}=3149.6 \pm 476.3 \mathrm{~mL} \cdot \mathrm{min}^{-1}\right)$ tests $(p<0.05)$.
The mean $\pm \mathrm{SD}$ values of tlim and $\mathrm{VO}_{2}$ slope during CWR and intermittent exercises for the PR and AR groups are presented in Table 2. A group vs. exercise condition interaction $\left(F=11.08, p=0.000, \eta^{2}=0.40\right)$ indicated longer tlim obtained during intermittent exercises (Short and Long) than CWR for both groups $(p<0.05)$. Considering the duration of the work and recovery type, tlim at Short was significantly longer than at Long only for the PR group ( $p<0.05)$. Group effect (i.e., PR vs. AR) was significant only when comparing the Short intermittent protocols $(p<0.05)$, with no significant difference for Long conditions $(p>0.05)$. There was a significant main effect for the exercise condition on $\mathrm{VO}_{2}$ slope values $(F=95.98, p<0.000$, $\left.\eta^{2}=0.90\right)$, but no group effect $\left(F=1.86, p=0.19, \eta^{2}=0.16\right)$ or interaction was detected $\left(F=0.02, p=0.99, \eta^{2}=0.01\right)$. $\mathrm{VO}_{2}$ slope was significantly greater at CWR than Short and Long conditions $(p<0.05)$.

The mean \pm SD values of $[\mathrm{La}]$ and HR during CWR and intermittent exercises for the PR and AR groups are presented in Table 3. There was a significant main effect for the exercise condition on $[\mathrm{La}]$ values $\left(F=4.72, p=0.01, \eta^{2}=0.22\right)$, but no effect of group $\left(F=0.05, p=0.81, \eta^{2}=0.04\right)$ or interaction was detected $\left(F=1.76 p=0.18, \eta^{2}=0.09\right)$. The [La] was significantly lower at Short than CWR and Long condition $(p<0.05)$. A group vs. exercise condition interaction $\left(F=5.00, p=0.01, \eta^{2}=0.23\right)$ indicated that HR was lower during Short than Long and CWR only for the $\mathrm{PR}$ group $(p<0.05)$. 
TABLE 2 | Mean \pm SD values of the time to exhaustion (tlim) and the slope of the oxygen uptake response (Slope) during the constant-work-rate (CWR) and intermittent exercise conditions (Short and Long), for passive (PR) and active (AR) recovery groups.

\begin{tabular}{|c|c|c|c|c|c|c|c|}
\hline & \multicolumn{3}{|c|}{$\mathrm{PR}(N=9)$} & \multicolumn{3}{|c|}{ AR $(N=9)$} & \multirow[t]{2}{*}{ Significance } \\
\hline & CWR & Short & Long & CWR & Short & Long & \\
\hline \multirow[t]{2}{*}{$\operatorname{tim}(s)$} & 540 & 1523 & 984 & 489 & 902 & 886 & ${ }^{\star} F=11.08$ \\
\hline & 116 & $411^{\ddagger, \dagger}$ & $260^{\ddagger}$ & 84 &  & $254^{\ddagger}$ & $p=0.000$ \\
\hline \multirow[t]{2}{*}{ Slope (L. $\left.\min ^{-2}\right)$} & 0.16 & 0.01 & 0.02 & 0.16 & 0.03 & 0.01 & ${ }^{++} F=5.34$ \\
\hline & 0.04 & $0.01^{\ddagger}$ & $0.01^{\ddagger}$ & 0.04 & $0.04^{\ddagger}$ & $0.01^{\ddagger}$ & $p=0.01$ \\
\hline
\end{tabular}

${ }^{*}$ Group vs. condition interaction; ${ }^{\ddagger} p<0.05$ relative to the CWR condition; ${ }^{\dagger} p<0.05$ relative to the Long condition; ${ }^{* *} p<0.05$ relative to the Short condition; ${ }^{++}$Main effect of exercise condition.

TABLE 3 | Mean \pm SD values of the blood lactate concentration ([La]) and heart rate (HR) during the constant-work-rate (CWR) and intermittent exercise conditions (Short and Long), for passive (PR) and active (AR) recovery groups.

\begin{tabular}{|c|c|c|c|c|c|c|c|}
\hline & \multicolumn{3}{|c|}{ PR } & \multicolumn{3}{|c|}{ AR } & \multirow[t]{2}{*}{ Significance } \\
\hline & CWR & Short & Long & CWR & Short & Long & \\
\hline \multirow[t]{2}{*}{ [La] (mM) } & 12.4 & 10.3 & 12.1 & 11.2 & 10.9 & 11.8 & ${ }^{++} F=4.72$ \\
\hline & 2.83 & 3.70 & 3.02 & 2.35 & 2.65 & 2.78 & $p=0.01$ \\
\hline $\mathrm{HR}$ (bpm) & 17714 & $17015^{\dagger}$ & 17711 & 1847 & 1836 & 1865 & ${ }^{*} F=5.00 p=0.01$ \\
\hline
\end{tabular}

${ }^{*}$ Group vs. condition interaction; ${ }^{\dagger} p<0.05$ relative to $C W R$ and Long conditions; ${ }^{++}$Main effect of exercise condition.

\section{DISCUSSION}

This, we believe, is the first study to compare the endurance performance and $\mathrm{VO}_{2} \mathrm{SC}$ kinetics during severe-intensity intermittent exercise performed with different durations and recovery types in active individuals. The data demonstrate that endurance performance during severe-intensity intermittent exercise is negatively influenced by active recovery only during shorter $(\sim 120 s)$ intermittent exercise. Interestingly, slopes describing the increases in $\mathrm{VO}_{2}$ with time (i.e., $\mathrm{VO}_{2} \mathrm{SC}$ ) and end-exercise $\mathrm{VO}_{2}$ were reduced during intermittent exercise (i.e., CWR vs. intermittent exercise). However, $\mathrm{VO}_{2}$ kinetics $\left(\mathrm{VO}_{2} \mathrm{SC}\right.$ and end-exercise $\left.\mathrm{VO}_{2}\right)$ were similar between work:recovery duration (short vs. long) and recovery type (passive vs. active) analyzed in the present study, therefore rejecting our original hypothesis. Thus, the relationship between $\mathrm{VO}_{2}$ kinetics $\left(\mathrm{VO}_{2} \mathrm{SC}\right.$ and end-exercise $\left.\mathrm{VO}_{2}\right)$ and endurance performance observed during CWR exercise (Jones et al., 2010; Barbosa et al., 2014a) seems to be differently regulated during severe-intensity intermittent exercise.

It has been widely reported that endurance performance during high-intensity intermittent exercise is improved when compared with CWR exercise (Demarie et al., 2000; Millet et al., 2003; Chidnok et al., 2012). However, both endurance performance and metabolic response are influenced by the characteristics of the protocol utilized during high-intensity intermittent exercise. Turner et al. (2006) analyzed the influence of duty cycle duration with the same work:recovery ratio $(10: 20 \mathrm{~s}, 30: 60 \mathrm{~s}, 60: 120 \mathrm{~s}$, and 90:180s) on pulmonary gas exchange and blood lactate dynamics during intermittent cycling exercise performed at $120 \% \mathrm{IVO}_{2}$ max. At this condition, a greater metabolic response (elevated blood lactate concentration and attainment of $\mathrm{VO}_{2} \mathrm{max}$ ) and exercise intolerance (i.e., subjects could not complete $30 \mathrm{~min}$ of exercise) were observed only for the longer duty cycles (i.e., 60:120s, and 90:180s). Although our intermittent exercise protocol presents different characteristics (e.g., work:recovery $=2: 1$ and exercise intensity $\left.=95 \% \mathrm{IVO}_{2} \max \right)$, it was also verified a reduced endurance performance during longer duty cycles performed with passive recovery. The intramuscular $\mathrm{PCr}$ concentration ([PCr]) kinetics both during and following high-intensity exercise presents a curvilinear profile and seems to be closely linked with $\mathrm{VO}_{2}$ kinetics (Rossiter et al., 2002). For instance, under the conditions of the present study, is very likely that the amplitude of [PCr] restoration during the $240 \mathrm{~s}$ recovery intervals (Long protocol) was not doubled than what was presented when 120 s periods of recovery (Short protocol) were allowed. Moreover, Chidnok et al. (2013) demonstrated that [PCr] restoration become longer as the intermittent protocol continued. Thus, [PCr] is progressively lower immediately before each repetition, particularly when duty-cycle duration is lengthened. The metabolites generated by muscle contraction at this condition, such as $\mathrm{Pi}, \mathrm{ADP}$, and AMP, increase glycolytic flux and consequently, glycolytic $\mathrm{H}^{+}$ (Adams et al., 1990; Conley et al., 1997) and lactate (Karpatkin et al., 1964) production. Low values of muscle [PCr] and $\mathrm{pH}$ (i.e., high values of $\left[\mathrm{H}^{+}\right]$) and consistently high values of $[\mathrm{Pi}]$ and [ADP] have been associated with fatigue development during high-intensity exercise (Jones et al., 2008; Vanhatalo et al., 2010).

Another factor that can influence both endurance performance and metabolic response is the activity pattern performed during the recovery intervals between each bout (Chidnok et al., 2012). Using the CP model, Chidnok et al. (2012) demonstrated that endurance performance during intermittent exercise was enhanced only when the recovery intervals were performed below CP. Active recovery performed below $\mathrm{CP}$ allows a partial $\mathrm{PCr}$ reconstitution and/or clearance of fatigue-related metabolites (Chidnok et al., 2013), with the former being apparently more important to enhance endurance performance during high-intensity intermittent exercise. Indeed, both endurance performance (Chidnok et al., 2012) and PCr reconstitution (Chidnok et al., 2013) are higher during intermittent exercise with passive recovery than during active recovery performed bellow $\mathrm{CP}$ condition. Thus, a lower PCr reconstitution can explain, at least in part, the impaired endurance performance during short condition performed with active recovery, as observed in the present study.

However, a different scenario emerges from the data obtained during the Long intermittent exercise protocol. At this condition, endurance performance was not modified by the active recovery periods. Two different mechanisms, which 
can occur simultaneously, could help explain this phenomenon. Firstly, the negative influence of active recovery on $\mathrm{PCr}$ reconstitution could be time-dependent, i.e., longer duty-cycle could allow more similar PCr reconstitution than a shorter one. The curvilinear PCr recovery profile supports this hypothesis (Harris et al., 1976). Secondly, the clearance of lactate and $\mathrm{H}^{+}$ ions within muscles might be higher during the longer dutycycle. A higher muscle $\mathrm{pH}$ can reduce, directly or indirectly (a more favorable metabolic milieu for $\mathrm{PCr}$ reconstitution), fatigue during high-intensity exercise. Alternatively, it is possible that $[\mathrm{PCr}]$ kinetics both during and following highintensity intermittent exercise would contribute progressively less to endurance performance when the duty-cycle duration is lengthened.

The end-exercise $\mathrm{VO}_{2}$ was not significantly different between CWR exercise and $\mathrm{VO}_{2}$ max measured during the incremental test. This is consistent with the fact that exhaustive exercise performed within the severe intensity domain (i.e., above $\mathrm{CP}$ ) is characterized by the development of the $\mathrm{VO}_{2} \mathrm{SC}$, which is truncated at $\mathrm{VO}_{2} \max$. Some interventional (e.g., endurance training and priming exercise) (Jones et al., 2007; Caritá et al., 2014) and correlational studies (Barbosa et al., 2014a) have produced evidences that both $\mathrm{VO}_{2}$ kinetics (a proxy for intramuscular PCr kinetics) (Rossiter et al., 2002) and $\mathrm{VO}_{2}$ max attainment is related to endurance performance during high-intensity exercise. Thus, it was hypothesized that $\mathrm{VO}_{2} \mathrm{SC}$ trajectory, which reflects the interaction between $\mathrm{VO}_{2} \mathrm{SC}$ and $\mathrm{VO}_{2}$ max attainment, could explain the endurance performance during high-intensity intermittent exercise. Indeed, it was demonstrated that $\mathrm{VO}_{2} \mathrm{SC}$ trajectory was faster during CWR exercise than during intermittent exercise, regardless of duration and recovery type. However, similar to the results found by Chidnok et al. (2012), $\mathrm{VO}_{2}$ SC trajectory was not significantly different among intermittent exercise, and endexercise $\mathrm{VO}_{2}$ was lower during these conditions than at CWR exercise. Thus, substrate utilization/accumulation, $\mathrm{VO}_{2}$ kinetics $\left(\mathrm{VO}_{2} \mathrm{SC}\right.$ trajectory and end-exercise $\left.\mathrm{VO}_{2}\right)$ and endurance performance during high-intensity exercise seem to present different relationship during CWR and intermittent exercise. Priming high-intensity exercise has previously been reported to reduce the amplitude of $\mathrm{VO}_{2} \mathrm{SC}$ and an increase in apparent $\mathrm{W}^{\prime}$ during subsequent exercise (Caritá et al., 2014, 2015; Dekerle et al., 2015). In this context, each preceding intermittent exercise bout may have "primed" the muscle (i.e., reduces the amplitude of $\mathrm{VO}_{2} \mathrm{SC}$ and/or raise the $\mathrm{W}^{\prime}$ ) during subsequent bouts. These modifications are consistent with enhanced endurance performance, and could help to explain the apparently different metabolic regulation imposed by the interaction between intervals duration and recovery type during intermittent exercise.

Our experimental protocol (i.e., exercise intensity, work:recovery durations and recovery types) was specifically designed to investigate the hypothetical association between intermittent endurance performance and $\mathrm{VO}_{2} \mathrm{SC}$ kinetics. Similar to previous studies (Caputo and Denadai, 2008; Barbosa et al., 2014a), both CWR and intermittent exercise were performed at $95 \% \mathrm{IVO}_{2}$ max. As demonstrated in the present study, exhaustive exercise performed at this intensity is characterized by the development of the $\mathrm{VO}_{2} \mathrm{SC}$ and $\mathrm{VO}_{2}$ max attainment. Some studies have utilized the "percentage delta" (for details please see Lansley et al., 2011) aiming to select a predetermined exercise intensity domain (i.e., heavy or severe) and/or to standardize the exercise intensity between subjects. Indeed, when compared to a more traditional method (e.g., $\% \mathrm{VO}_{2} \mathrm{max}$ ), this approach allows a lower inter-subject variability of physiological responses to CWR exercise (Lansley et al., 2011). However, for the first time, the present study have normalized the wok:recovery durations based on the individual $\mathrm{VO}_{2} \mathrm{SC}$ kinetics response. Thus, we are confident that the inter-subject variability of physiological responses during the intermittent exercise was attenuated. Finally, this study presented a possible limitation, since the effect of passive and active recovery on intermittent exercise was analyzed using 2 different groups of active individuals. Hypothetically, this experimental design could be influenced by the individual variability on both endurance performance and $\mathrm{VO}_{2} \mathrm{SC}$ kinetics. However, $\mathrm{PR}$ and $\mathrm{AR}$ groups have presented similar data during incremental $\left(\mathrm{VO}_{2} \max , \mathrm{IVO}_{2} \max , 95 \% \quad \mathrm{IVO}_{2} \mathrm{max}\right.$ and $\mathrm{LT}$ ) and CWR exercise (endurance performance and $\mathrm{VO}_{2} \mathrm{SC}$ kinetics). Therefore, the possibility of inter-subject variability influencing the recovery types comparisons was probably reduced. This limitation comes from the heavy testing required to be undertaken by each subject to test our research hypothesis. It is important to note that a short-term training program (6 sessions) involving high-intensity exercise (repeated all-out sprint training) have reduced the amplitude of the $\mathrm{VO}_{2} \mathrm{SC}$ and increased tolerance to high-intensity exercise in recreationally active subjects (Bailey et al., 2009). Thus, if a repeated measures design has been utilized in our experimental approach, a confounding factor could be added to our analysis, since the volunteers would have to perform 6 bouts of severe-intensity exercise.

\section{CONCLUSION}

The present study showed that under our experimental conditions (i.e., exercise intensity, work:recovery durations and recovery type), intermittent exercise enhances endurance performance during severe-intensity exercise, independently of intervals duration and recovery type. Passive recovery is superior in relation to active recovery to enhance endurance performance only during shorter duty-cycles. Although $\mathrm{VO}_{2} \mathrm{SC}$ trajectory is attenuated during high-intensity intermittent exercise, its alteration does not seem to explain the interaction effects of intervals duration and recovery type on endurance performance. Moreover, the end-exercise $\mathrm{VO}_{2}$ was lower during intermittent exercise than at CWR exercise. Thus, severe-intensity intermittent exercise performed with different intervals duration and recovery type seems to modify the relationship between endurance performance and $\mathrm{VO}_{2}$ kinetics observed during CWR exercise. Further studies using a repeated measures design are required to examine the effect of severe-intensity intermittent exercise on both endurance performance and $\mathrm{VO}_{2} \mathrm{SC}$ in trained individuals. A threshold 
in the duration of the recovery, from which $\mathrm{PCr}$ resynthesis and/or W' reconstitution would be less affected by active recovery could be identified. This can help to explain and confirm our main results, giving support to elaborate a more sophisticate interval training programs for different populations.

\section{AUTHOR CONTRIBUTIONS}

Study design: BD and CG. Data acquisition and analysis: LB, BD, and CG and Writing the paper: $\mathrm{LB}, \mathrm{BD}$, and CG.

\section{REFERENCES}

Adami, A., Sivieri, A., Moía, C., Perini, R., and Ferretti, G. (2013). Effects of step duration in incremental ramp protocols on peak power and maximal oxygen consumption. Eur. J. Appl. Physiol. 113, 2647-2653. doi: 10.1007/s00421-0132705-9

Adams, G. R., Foley, J. M., and Meyer, R. A. (1990). Muscle buffer capacity estimated from $\mathrm{pH}$ changes during rest-to-work transitions. J. Appl. Physiol. 69, 968-972.

Bailey, S. J., Wilkerson, D. P., Dimenna, F. J., and Jones, A. M. (2009). Influence of repeated sprint training on pulmonary $\mathrm{O} 2$ uptake and muscle deoxygenation kinetics in humans. J. Appl. Physiol. 106, 1875-1887. doi: 10.1152/japplphysiol. 00144.2009

Barbosa, L. F., Greco, C. C., and Denadai, B. S. (2014a). The slope of the VO2 slow component is associated with exercise intolerance during severe-intensity exercise. Acta Physiol. Hung. 101, 517-523. doi: 10.1556/APhysiol.101.2014.4.13

Barbosa, L. F., Montagnana, L., Denadai, B. S., and Greco, C. C. (2014b). Reliability of cardiorespiratory parameters during cycling exercise performed at the severe domain in active individuals. J. Strength Cond. Res. 28, 976-981. doi: 10.1519/ JSC.0b013e3182a1f408

Bentley, D. J., and McNaughton, L. R. (2003). Comparison of W(peak), VO2(peak) and the ventilation threshold from two different incremental exercise tests: relationship to endurance performance. J. Sci. Med. Sport 6, 422-435. doi: 10. 1016/S1440-2440(03)80268-2

Caputo, F., and Denadai, B. S. (2008). The highest intensity and the shortest duration permitting attainment of maximal oxygen uptake during cycling: effects of different methods and aerobic fitness level. Eur. J. Appl. Physiol. 103, 47-57. doi: 10.1007/s00421-008-0670-5

Caritá, R. A., Greco, C. C., and Denadai, B. S. (2014). The positive effects of priming exercise on oxygen uptake kinetics and high-intensity exercise performance are not magnified by a fast-start pacing strategy in trained cyclists. PLOS ONE 9:e95202. doi: 10.1371/journal.pone.0095202

Caritá, R. A., Greco, C. C., and Denadai, B. S. (2015). Prior heavy-intensity exercise's enhancement of oxygen-uptake kinetics and short-term highintensity exercise performance independent of aerobic-training status. Int. J. Sports Physiol. Perform. 10, 339-345. doi: 10.1123/ijspp.2014-0131

Chidnok, W., Dimenna, F. J., Bailey, S. J., Vanhatalo, A., Morton, R. H., Wilkerson, D. P., et al. (2012). Exercise tolerance in intermittent cycling: application of the critical power concept. Med. Sci. Sports Exerc. 44, 966-976. doi: 10.1249/MSS. 0b013e31823ea28a

Chidnok, W., DiMenna, F. J., Fulford, J., Bailey, S. J., Skiba, P. F., Vanhatalo, A., et al. (2013). Muscle metabolic responses during high-intensity intermittent exercise measured by (31)P-MRS: relationship to the critical power concept. Am. J. Physiol. Regul. Integr. Comp. Physiol. 305, R1085-R1092. doi: 10.1152/ ajpregu.00406.2013

Conley, K. E., Blei, M. L., Richards, T. L., Kushmerick, M. J., and Jubrias, S. A. (1997). Activation of glycolysis in human muscle in vivo. Am. J. Physiol. Cell Physiol. 273, C306-C315.

Dekerle, J., de Souza, K. M., de Lucas, R. D., Guglielmo, L. G., Greco, C. C., and Denadai, B. S. (2015). Exercise tolerance can be enhanced through a

\section{FUNDING}

Supported by Fundação de Amparo à Pesquisa do Estado de São Paulo (FAPESP) (grant 2009/07700-2 and grant 2016/22907-6), Conselho Nacional de Desenvolvimento Científico e Tecnológico (CNPq) and Fundação para o Desenvolvimento da Unesp (FUNDUNESP).

\section{ACKNOWLEDGMENTS}

The authors appreciate the time and effort expended by all volunteer subjects in this study.

change in work rate within the severe intensity domain: work above critical power is not constant. PLoS ONE. 10:e0138428. doi: 10.1371/journal.pone.01 38428

Demarie, S., Koralsztein, J. P., and Billat, V. (2000). Time limit and time at VO2max during a continuous and an intermittent run. J. Sports Med. Phys. Fitness 40, 96-102.

Fukuba, Y., Miura, A., Endo, M., Kan, A., Yanagawa, K., and Whipp, B. J. (2003). The curvature constant parameter of the power-duration curve for variedpower exercise. Med. Sci. Sports Exerc. 35, 1413-1418. doi: 10.1249/01.MSS. 0000079047.84364.70

Harris, R. C., Edwards, R. H., Hultman, E., Nordesjö, L. O., Nylind, B., and Sahlin, K. (1976). The time course of phosphorylcreatine resynthesis during recovery of the quadriceps muscle in man. Pflügers Arch. 367, 137-142. doi: 10.1007/ BF00585149

Hwang, C. L., Wu, Y. T., and Chou, C. H. (2011). Effect of aerobic interval training on exercise capacity and metabolic risk factors in people with cardiometabolic disorders: a meta-analysis. J. Cardiopulm. Rehabil. Prev. 31, 378-385. doi: 10. 1097/HCR.0b013e31822f16cb

Jones, A. M., Fulford, J., and Wilkerson, D. P. (2008). Influence of prior exercise on muscle [phosphorylcreatine] and deoxygenation kinetics during high-intensity exercise in men. Exp. Physiol. 93, 468-478. doi: 10.1113/expphysiol.2007. 041897

Jones, A. M., Grassi, B., Christensen, P. M., Krustrup, P., Bangsbo, J., and Poole, D. C. (2011). Slow component of VO2 kinetics: mechanistic bases and practical applications. Med. Sci. Sports Exerc. 43, 2046-2062. doi: 10.1249/MSS. 0b013e31821fcfc1

Jones, A. M., Vanhatalo, A., Burnley, M., Morton, R. H., and Poole, D. C. (2010). Critical power: implications for determination of VO2max and exercise tolerance. Med. Sci. Sports Exerc. 42, 1876-1890. doi: 10.1249/MSS. 0b013e3181d9cf7f

Jones, A. M., Wilkerson, D. P., Berger, N. J., and Fulford, J. (2007). Influence of endurance training on muscle [PCr] kinetics during high-intensity exercise. Am. J. Physiol. Regul. Integr. Comp. Physiol. 293, R392-R401. doi: 10.1152/ ajpregu.00056.2007

Karpatkin, S., Helmreich, E., and Cori, C. F. (1964). Regulation of glycolysis in muscle. II. Effect of stimulation and epinephrine in isolated frog sartorius muscle. J. Biol. Chem. 239, 3139-3145.

Lamarra, N., Whipp, B. J., Ward, S. A., and Wasserman, K. (1987). Effect of interbreath fluctuations on characterizing exercise gas exchange kinetics. $J$. Appl. Physiol. 62, 2003-2012.

Lansley, K. E., Dimenna, F. J., Bailey, S. J., and Jones, A. M. (2011). A 'new' method to normalise exercise intensity. Int. J. Sports Med. 32, 535-541. doi: 10.1055/s0031-1273754

Laursen, P. B., and Jenkins, D. G. (2002). The scientific basis for highintensity interval training: optimising training programmes and maximising performance in highly trained endurance athletes. Sports Med. 32, 53-73. doi: 10.2165/00007256-200232010-00003

Marsh, A. P., and Martin, P. E. (1997). Effect of cycling experience, aerobic power, and power output on preferred and most economical cycling cadences. Med. Sci. Sports Exerc. 29, 1225-1232. doi: 10.1097/00005768-19970900000016 
Millet, G. P., Candau, R., Fattori, P., Bignet, F., and Varray, A. (2003). VO2 responses to different intermittent runs at velocity associated with $\mathrm{VO} 2 \mathrm{max}$. Can. J. Appl. Physiol. 28, 410-423. doi: 10.1139/h03-030

Murgatroyd, S. R., Ferguson, C., Ward, S. A., Whipp, B. J., and Rossiter, H. B. (2011). Pulmonary O2 uptake kinetics as a determinant of high-intensity exercise tolerance in humans. J. Appl. Physiol. 110, 1598-1606. doi: 10.1152/ japplphysiol.01092.2010

Poole, D. C., Ward, S. A., Gardner, G. W., and Whipp, B. J. (1988). Metabolic and respiratory profile of the upper limit for prolonged exercise in man. Ergonomics. 31, 1265-1279. doi: 10.1080/00140138808966766

Roffey, D. M., Byrne, N. M., and Hills, A. P. (2007). Effect of stage duration on physiological variables commonly used to determine maximum aerobic performance during cycle ergometry. J. Sports Sci. 25, 1325-1335. doi: 10.1080/ 02640410601175428

Rossiter, H. B., Ward, S. A., Kowalchuk, J. M., Howe, F. A., Griffiths, J. R., and Whipp, B. J. (2002). Dynamic asymmetry of phosphocreatine concentration and $\mathrm{O}(2)$ uptake between the on- and off-transients of moderate- and highintensity exercise in humans. J. Physiol. 541, 991-1002. doi: 10.1113/jphysiol. 2001.012910

Turner, A. P., Cathcart, A. J., Parker, M. E., Butterworth, C., Wilson, J., and Ward, S. A. (2006). Oxygen uptake and muscle desaturation kinetics during intermittent cycling. Med. Sci. Sports Exerc. 38, 492-503. doi: 10.1249/01.mss. 0000188450.82733.f0
Vanhatalo, A., Fulford, J., DiMenna, F. J., and Jones, A. M. (2010). Influence of hyperoxia on muscle metabolic responses and the power-duration relationship during severe-intensity exercise in humans: a $31 \mathrm{P}$ magnetic resonance spectroscopy study. Exp. Physiol. 95, 528-540. doi: 10.1113/expphysiol.2009. 050500

Whipp, B. J., and Rossiter, H. B. (2005). "The kinetics of oxygen uptake: physiological inferences from the parameters," in Oxygen Uptake Kinetics in Sport, Exercise, and Medicine, ed A. M. Jones and D. C. Poole (London: Routledge), 64-94.

Zoladz, J. A., Rademaker, A. C., and Sargeant, A. J. (1995). Non-linear relationship between $\mathrm{O} 2$ uptake and power output at high intensities of exercise in humans. J. Physiol. 488, 211-217. doi: 10.1113/jphysiol.1995.sp020959

Conflict of Interest Statement: The authors declare that the research was conducted in the absence of any commercial or financial relationships that could be construed as a potential conflict of interest.

Copyright (c) 2016 Barbosa, Denadai and Greco. This is an open-access article distributed under the terms of the Creative Commons Attribution License (CC BY).

The use, distribution or reproduction in other forums is permitted, provided the original author(s) or licensor are credited and that the original publication in this journal is cited, in accordance with accepted academic practice. No use, distribution or reproduction is permitted which does not comply with these terms. 